\title{
Conjugate Heat Transfer of an Internally Air-Cooled Nozzle Guide Vane and Shrouds
}

\author{
Lei-Yong Jiang, Yinghua Han and Prakash Patnaik
}

Additional information is available at the end of the chapter

http://dx.doi.org/10.5772/intechopen.74104

\begin{abstract}
The accurate evaluation of the aero-thermodynamic working environments of gas turbine critical components is essential in the development of advanced gas turbine engines, such as cooling flow arrangement and service life assessment. In this chapter, as a technology demonstration, the conjugate heat transfers of the internally air-cooled nozzle guide vane (NGV) and shrouds of a gas turbine engine at flight conditions are numerically examined. The simulations are performed with a high-fidelity CFD model and adequately defined boundary conditions. The effect of the non-dimensional distance from wall surfaces, $y+$, on the wall temperature is studied. The characteristics of the complex three-dimensional flow and temperature fields are revealed, and the heat fluxes between the hot gas, NGV body, and cooling airflow at selected cross sections are presented and discussed. It is clear that the traditional one-dimensional semiempirical approach is no longer suitable. Generally, the Mach number is higher, and the temperature is lower on the NGV suction side than on the pressure side. It is found that two high-temperature zones occur on the NGV pressure side and the temperature over the middle section is relatively low. These findings are related to where the cooling holes and outlets are located and consistent with the field observation of NGV damages.
\end{abstract}

Keywords: conjugate heat transfer, gas turbine, nozzle guide vane, internal air cooling

\section{Introduction}

Conjugate heat transfer (CHT) means the combined heat transfer processes between and in solid and fluid, where conduction usually dominates in solid and convection generally governs in fluid. The CHT analysis is based on solid physics and governed by a set of mathematical differential equations with well-defined boundaries. 
The CHT analysis has now become an essential tool in the development and maintenance of thermal systems from aerospace, telecommunication, medicine, and atmosphere/ocean interaction to food processing. It can provide realistic 3D heat transfer analyses for practical complex devices, and this is why it has been extensively used in a wide range of applications to replace the traditional 1D semiempirical approach in CHT analyses.

In this chapter, as a technology demonstration, the conjugate heat transfer of the internally air-cooled nozzle guide vane (NGV) and shrouds of a gas turbine engine at flight conditions is numerically analyzed. Due to the continuous demand for high efficiency [1], the NGV is always exposed to high-pressure and high-temperature working environments and the field variables surrounding and inside the NGV change drastically. A higher temperature reduces the fatigue strength of the material, while a larger temperature gradient increases the thermal strain excursions and causes higher stresses. Both of them can reduce the NGV fatigue life. Therefore, for reliable life analysis of engine critical components, accurately assessing their aerothermodynamic working environments is essential.

Due to the high-pressure and high-temperature conditions, experimental measurements are particularly difficult inside the gas turbine engine. Computational fluid dynamics (CFD) analyses are normally used and continuously validated for calculating the aero-thermal loads for gas turbine engines. Mazur and coworkers [2] performed the conjugate heat transfer analysis between the NGV/shrouds and flow for a power generation gas turbine combustor. They found that based on the calculated thermal loads, stress, and creep strain analyses, it was feasible to assess the nozzle remaining lifetime. However, the shroud thermal boundary conditions were not given in their paper. Charbonnier and coauthors [3] validated the heat transfer predictions with the measured data for a film-cooled linear cascade. They found that the CFD predictions were in good agreement with the measurements of the vane surface Mach number, film cooling row discharge coefficients, wall heat flux, and adiabatic film cooling effectiveness. A conjugate heat transfer solver was developed by Heidmann and coworkers [4], where no grid was required inside the solid regions. They used this code to study the heat transfer of the middle span section of a film-cooled turbine vane with a few blade materials. The results indicated that the heat transfer in a film-cooled vane was complex and the simple 1D conduction analysis from the vane surface to the plenum surface was no longer valid.

In spite of being a common practice in the industry, the detailed CFD analysis of internally air-cooled nozzle guide vanes and shrouds with high-fidelity geometry and well-defined boundary conditions is rare. There may be three reasons. Firstly, the realistic geometry of realworld turbine NGV/shrouds is complex, particularly for the internal cooling flow channels. Secondly, fine mesh near the walls is required in order to accurately calculate heat transfer [5], and thus the mesh size can be considerably large even for a single NGV and shrouds. Thirdly, it can be difficult to find reliable boundary conditions, where both the engine combustor flow field and secondary air-cooling flow have to be considered. The significant effect of secondary air-cooling flow on the end walls of an air-film-cooled NGV was found by Harsqama and coworkers [6].

As a technology demonstration, a numerical investigation has been performed to study the conjugate heat transfer of the internally air-cooled nozzle guide vane of a practical gas 
turbine engine at flight conditions. The CFD model with high-fidelity geometry, complex flow features, temperature contours around/inside the NGV, and heat fluxes at typical NGV cross sections are presented and described. Finally, a number of highlights are given.

\section{High-fidelity CFD model of air-cooled NGV}

\subsection{Computational domain and mesh of internally air-cooled NGV and shrouds}

A conventional can-annular gas turbine combustor is chosen for this study. An annular chamber accommodates 6 combustion cans, and 60 NGVs are located at the exit of the combustor. The high-fidelity geometry of the NGV and shrouds is retained in the present work. For example, the curved three-dimensional internal/external surfaces are maintained.

Figure 1 illustrates the computational domain and mesh of an internally air-cooled NGV with internal and external shrouds. It is a $6^{\circ}$ sector in the engine combustor coordinates, and the periodic boundaries of the geometry are rotated twice to avoid cutting through the NGV body. The flow domain starts upstream of the flat plates of the internal and external shrouds and is extended downstream of the shrouds by half of the NGV chord length. Both flow and solid meshes are shown in Figure 1(a), and in Figure 1(b), only the solid mesh is illustrated. The mesh at one middle cross section is given in Figure 1(c), where the thick black lines represent the NGV body cross section which separates the flow and solid region meshes. In order to properly solve the conjugate heat transfer between the fluid and solid regions, fine grids were created inside the NGV body and flow regions around the NGV. The NGV CAD geometry file was generated based on laser-scanned cloud of points, which resulted in difficulties in mesh generation. A number of meshes were generated and attempt runs were carried out to improve numerical simulation quality. Eventually, a mesh size of 6.3 million cells was used for all simulations. Fine grids were also created in the vicinity of the NGV internal and external walls, fins, holes, and cooling air slot, as shown in Figure 1(b). In the flow regions away from the NGV walls, coarse nodes were generated. At the flight conditions, the nondimensional wall boundary parameter, $y+$ was in a range of $\sim 0-25$ on the cooling slot, fins, and NGV internal walls. It varied from 5 to 35 at the NGV external walls and from 5 to 130 at the shroud walls.

As illustrated in Figure 1(a), and 1(b), the hot gas mixture, the main flow, from the engine combustor runs over the NGV body. The cooling air coming from the combustor annular chamber enters the NGV thin-wall cooling slot through the cooling air inlet above the flat plate of the internal shroud, travels through six holes facing the NGV leading edge and one hole looking downstream into the cooling chamber inside the NGV body, then flows out of the NGV body at the four rectangular air exits, merges together with the main flow, and finally exits the flow domain. There are twelve cooling fins on either side of the cooling chamber to increase the heat transfer between the cooling air and NGV internal surfaces. The cooling air in the NGV body absorbs heat from the NGV internal surfaces and decreases its external surface temperature through impingement and convection. 

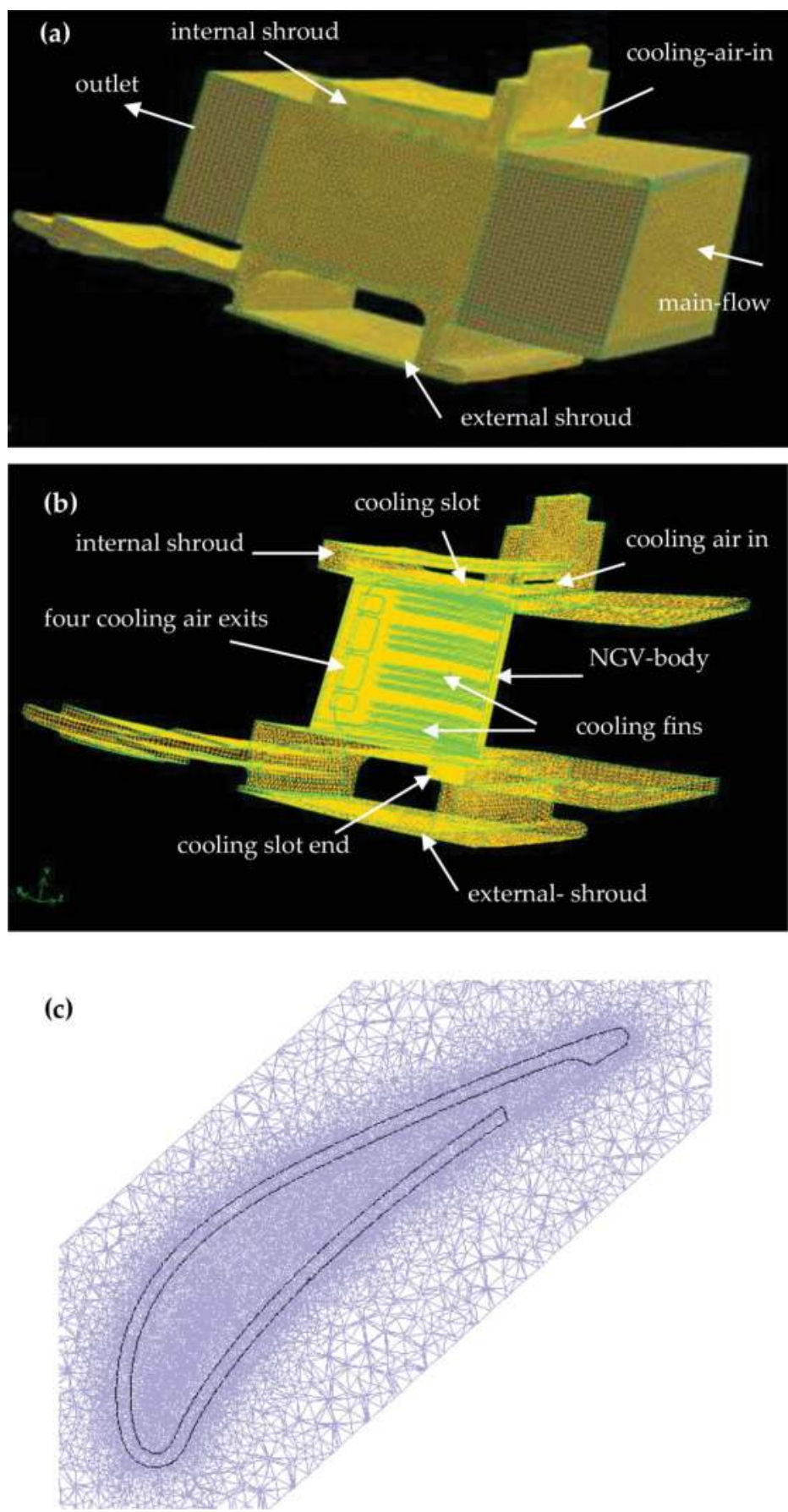

Figure 1. Computational domain and mesh: (a) flow and solid mesh, (b) solid mesh, and (c) mesh at one middle cross section. 


\begin{tabular}{lllll}
\hline Case & Size & $\mathbf{y}^{+}$ & $\Delta \mathbf{t}$ & $\Delta \mathbf{t} / \mathbf{t}$ \\
\hline Mesh-1 & 21,200 & $\sim 200$ & 14.9 & $1.3 \%$ \\
Mesh-2 & 282,000 & $\sim 60$ & 8.0 & $0.7 \%$ \\
Mesh-3 & 180,000 & $\sim 10$ & 9.7 & $0.8 \%$ \\
Mesh-4 & 265,200 & $\sim 0.5$ & $\sim 0$ & $\sim 0$ \\
\hline
\end{tabular}

Table 1. Variation of hot wall temperature with $y+$ value.

As indicated in Figure 1, the high-fidelity geometry of the NGV and shrouds is maintained in the present work. For example, the curved 3D internal/external surfaces between the NGV body and shrouds are retained.

\subsection{The effect of the nondimensional distance from the wall $(y+)$ on the wall temperature}

The main objective of this work is to adequately compute conjugate heat transfer among the external combustion mixture, NGV body, and internal cooling airflow. Therefore, the mesh independence effect in the numerical solutions is examined by simplified 2D simulations. The computational domain of these 2D simulations contains a flat metal plate, and its thickness is equal to the mean value of the NGV body. A hot gas flow with the velocity and temperature corresponding to the combustion mixture flows over one side of the plate, and a cooling flow with the velocity and temperature equal to those in the NGV simulation, runs along the other side of the plate.

The mesh size and result of four test cases are summarized in Table 1, where rectangular cells are generated for all meshes. In Mesh-1 and Mesh-2, grids are distributed uniformly in both coordinates, while for Mesh-3 and Mesh-4, in order to maintain reasonable mesh size, fine grids are created near the walls and gradually expand away from the walls.

As indicated in Table 1, the hot wall temperature for $\mathrm{y}+\approx 0.5$ (Mesh-4) is taken as the best result, $\Delta t$ represents the deviation of wall temperature from the Mesh- 4 case, and $\Delta t / t$ denotes the difference in wall temperature between Mesh 4 and other cases normalized by that of the Mesh- 4 case. The comparisons suggest that in the range of $y+$ values (0.5-200), the maximum deviation is limited to about $1.3 \%$. The temperature values of $\Delta \mathrm{t}$ and $\Delta \mathrm{t} / \mathrm{t}$ at the cooling side wall are almost identical as those in Table 1, and thus they are not presented here. As stated before, $y+$ varies from $\sim 0$ to 35 at the NGV external walls and in a range of 5-130 at the shroud walls in the present work, and thus the mesh used in the simulations is fine enough for wall temperature calculation.

\section{Numerical simulations}

In the present study, steady compressible turbulent thermal flow fields are resolved. A commercial software code, ANSYS Fluent, is applied to all the numerical simulations. In the following subsections, the numerical approach, boundary conditions, and solution methods are discussed briefly. 


\subsection{Numerical approach}

The Favre-averaged conservation differential equations for mass, momentum, and energy can be readily found in the literature, such as $[7,8]$, and thus they are not presented here. The viscous heating and radiation heat transfer items in the energy equation are not considered in the present study since their effect on the flow field around the NGV is negligibly small.

The SST k- $\omega$ model (the shear-stress transport model) is selected to model turbulent transfer items in the flow. Major improvements are observed in the prediction of separation and adverse pressure gradient flows in comparison with the standard $k-\omega$ and $k-\varepsilon$ models [9]. This model has been successfully used in the heat transfer simulation for the film cooling of a linear cascade, and the results are in good agreement with the experimental measurements [3].

In engineering applications, it is difficult to keep y + values to a prescribed value for all walladjacent cells throughout the domain for complex devices. To keep numerical solutions insensitive to wall mesh, an automatic wall treatment is formulated for the SST model in ANSYS Fluent [8]. According to local y + values, it shifts automatically from the viscous sub-layer treatment to the fully turbulent region formulation, while in the buffer layer between the viscous sub-layer and fully turbulent region, a blending function is used.

In the present work, the thermal properties of air are utilized for the combustion gas mixture because the overall fuel-air ratio in gas turbine combustors is low [1]. The polynomials as a function of temperature are applied to compute the specific heat of air. The molecular viscosity and thermal conductivity of air at the average temperature over the whole domain are used in simulations. The polynomials are also used to compute the specific heat and conductivity for Hastelloy $\mathrm{X}$ alloy.

\subsection{Boundary conditions}

The boundary conditions at the inlet are taken from a previous benchmark simulation of the gas turbine combustor at the flight conditions. The simulation is similar to those carried out in [10], where the two-phase, compressible, reacting flow of a $60^{\circ}$ sector of the can-annular combustor with ten NGVs (no internal cooling flow passages) attached is resolved. The average total temperature and pressure obtained from this simulation at the matching location are defined as the inlet boundary conditions for the main flow (see Figure 1). As stated earlier, the NGV cooling air comes from the annular chamber of the combustor. The average temperature and pressure values at the ten NGV cooling air slots from the simulation are specified as the cooling air inlet conditions for the cooling flow (see Figure 1). The cooling airflow rate is $2.3 \%$ of the total inlet airflow. To consider the effect of the flow tangential velocity on the pressure distribution, a radial equilibrium pressure distribution is applied to the domain exit plane. The Reynolds number calculated with the chord length and inlet flow conditions is $1.0 \times 10^{5}$.

The thermal boundary conditions on the shroud outer surfaces are estimated from the secondary airflow information (flow temperature and Mach number) of [11]. A 2D simulation is carried out, and in this simulation, the stream-wise cross sections of the internal/external shrouds remain the same, the NGV body is removed from the computational domain, and 
two additional flow zones beside the external and internal shrouds are included in the domain. The wall temperatures obtained from the simulation are utilized at the outer surfaces of the NGV/shroud assembly. The turbulence kinetic energy and specific dissipation rate at the inlets of the $\mathrm{NGV} /$ shroud assembly are calculated with an estimated turbulence intensity of $10 \%$ and inlet hydraulic diameters. At the side boundaries of the $6^{\circ}$ sector, periodic boundaries are specified.

\subsection{Solution methods}

A pressure-based coupled solver with a second-order accuracy scheme is used to solve the flow field. At convergence, the normalized residuals are less than $4 \times 10^{-5}$ for all velocity components and energy and around $6 \times 10^{-4}$ for turbulent parameters. The mass flow rate monitored at the four rectangular outlets of the internal cooling airflow remains unchanged at least for the first three digits. All these ensure that the simulation reaches steady conditions. A 4-node LINUX cluster, with 64-bit, $2.6 \mathrm{GHz}$, 8-core, and 64 GB RAM per node, is used to perform all the simulations.

\section{Results and discussion}

In the following subsections, some of the simulation results around and inside the NGV/ shrouds at the flight conditions are given and discussed.

\subsection{Flow field of the NGV/shrouds}

The Mach number contours at the section passing through an upstream hole and the downstream hole of the cooling airflow passage are shown in Figure 2. Figure 2(a) represents the whole cross section, and Figure 2(b) is a plot zoomed near the NGV. The temperature contours at the same cross section are illustrated in Figure 3, and the local temperature contours around the NGV are enlarged in Figure 3(b). In this figure and other figures in the chapter, the temperatures are normalized by the maximum temperature in the computational domain. Lines in the plots denote the NGV and internal cooling slot metal walls.

Figures 2 and 3 clearly display the compressible flow features. In the inlet section, the flow velocity and temperature are more or less constant. The flow acts noticeably differently on the pressure and suction sides as it approaches the NGV. A stagnation point is formed the NGV leading edge of the pressure side, as shown in Figure 2. After the leading edge, the flow gradually accelerates, and the Mach number or velocity remains relatively low till reaching the trailing edge. More complex interactions among the main flow, cooling flow, and NGV geometry happen near the rectangular opening. The flow rapidly speeds up from the end edge of the rectangular opening, and behind the trailing edge, a small recirculation or stagnation zone is observed. The static temperature at the pressure side is relatively high except for the small downstream region, as shown in Figure 3, and this is because the flow velocity is relatively low except for the small region from the end of the rectangular opening to the trailing edge. 
(a)
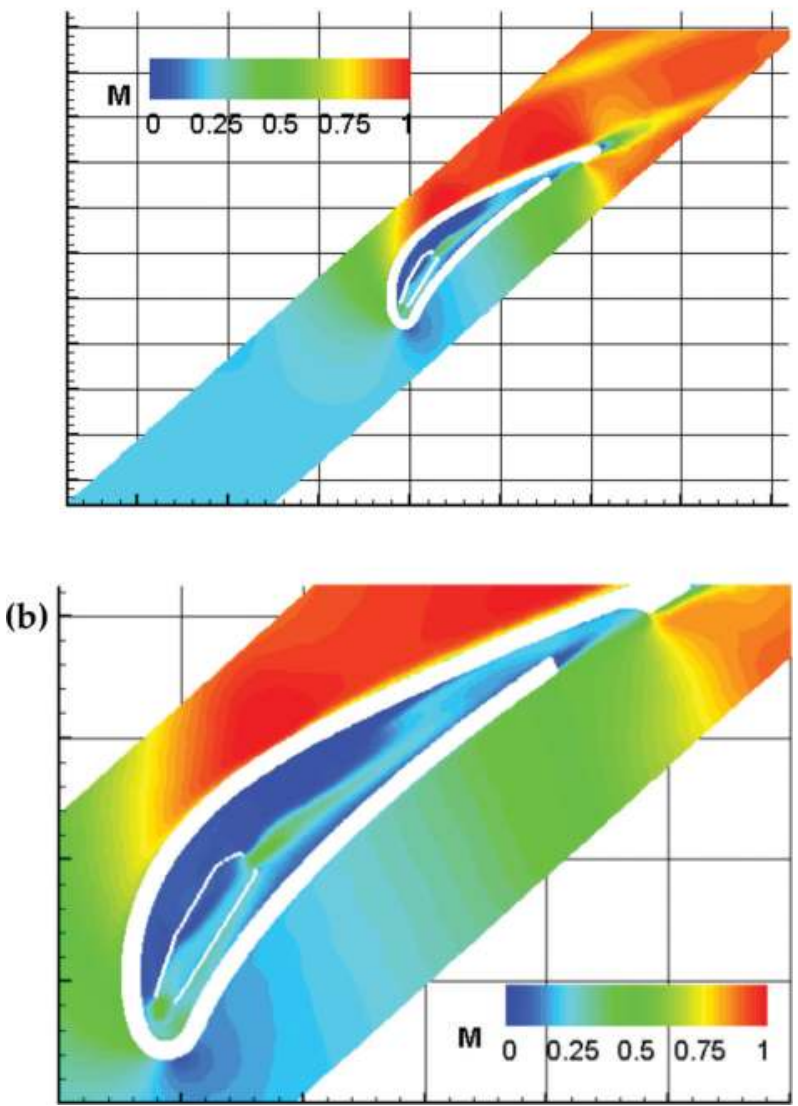

Figure 2. Mach number contours at the section across an upstream hole and the downstream hole of the cooling slot. (a) the whole section and (b) the zoomed local region.

In contrast, the flow velocity accelerates quickly along the suction surface after the leading edge, stays high in most of the downstream region, and rapidly decelerates at the trailing edge. The maximum Mach number is slightly higher than 1.0 in the flow, and two local supersonic spots occur in Figure 2. Because of the rapid flow acceleration along the suction side, the temperature drops quickly from the leading edge and remains relatively the same level at most downstream regions. In short, as illustrated in Figure 2, the pressure side wall is subject to higher temperature than the suction side wall, which agrees the observation in [2].

At this section in the cooling chamber, the flow velocity is fairly high around the leading edge, middle, and downstream portion. However, the cooling air velocity is low in a large region around the suction side. It is anticipated that the cooling chamber and fin geometries are the reasons for these observations. 
(a)

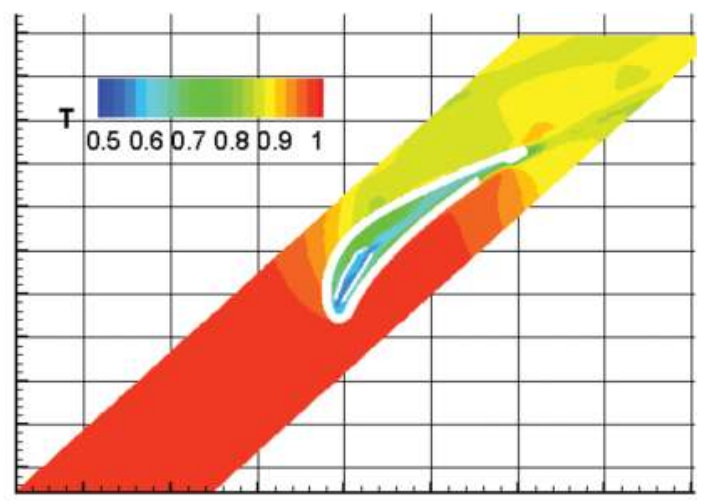

(b)

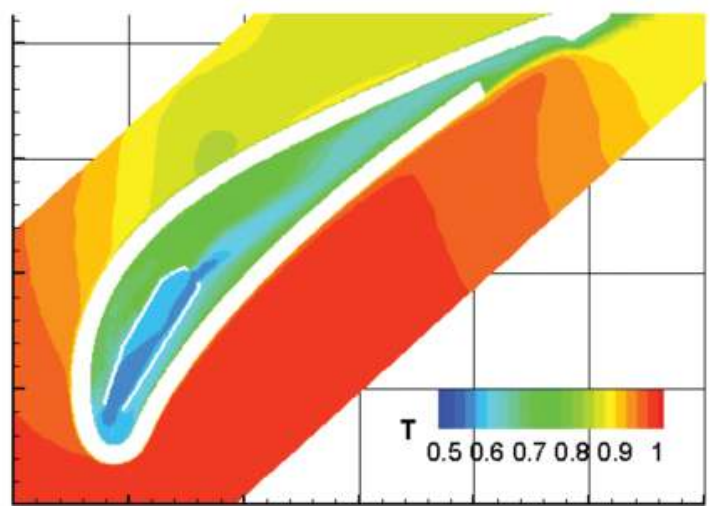

Figure 3. Temperature contours at the section across an upstream hole and the downstream hole of the cooling slot. (a) the whole section and (b) the zoomed local region.

The velocity vector plot is presented in Figure 4 at the same cross section as Figures 2-3. Figure 4(a) shows a whole picture of velocity vectors around the NGV section wall, Figure 4(b) displays vector details around the upstream cooling hole of the cooling slot, and Figure 4(c) is a plot zoomed around the downstream hole. The vector length indicates its magnitude, and the color stands for its Mach number. For the present design, both impingement and convection cooling are utilized to cool the NGV walls. As shown in Figure 4(a) and (b), the cooling air directly impacts on the inner surface of the NGV near the leading edge. In all three plots of Figure 4 the convection cooling is clearly observed, i.e., the cooling air runs along the NGV external/internal surfaces.

Table 2 gives the mass flow rate through each cooling hole as a percentage of the total cooling air. The upstream holes in Table 2 are listed as 1 to 6 beginning from the internal shroud side. At the four upstream holes (3-6) and the downstream hole, the mass flow rates are at the same level, and at upstream holes $1-2$, the mass flow rates are close to each other. The maximum difference in the cooling flow rate is $3.2 \%$ between holes 2 and 6 . 
(a)

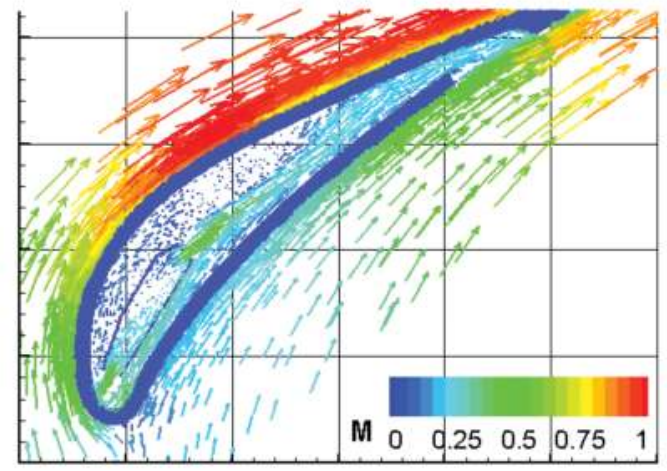

(b)

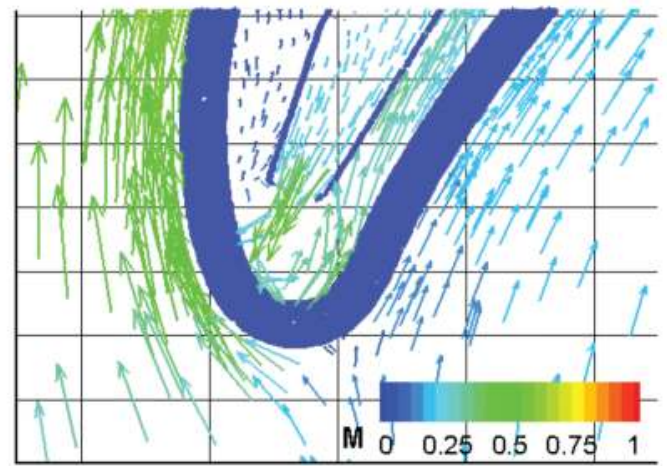

(c)

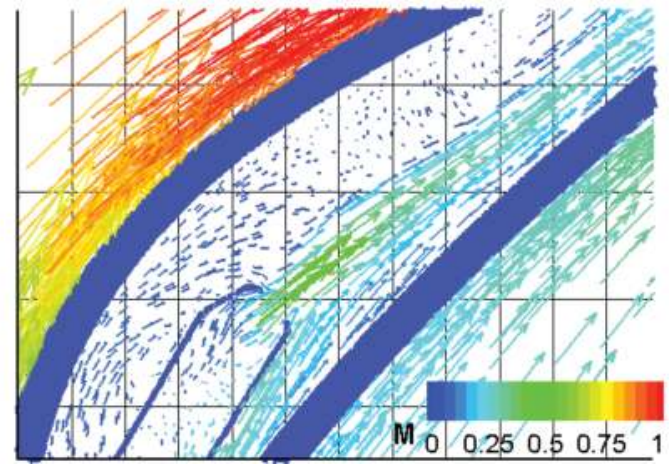

Figure 4. Velocity vectors colored by Mach number levels: (a) the section across an upstream hole and the downstream hole, (b) the section across an upstream hole, and (c) the section across the downstream hole.

\subsection{Temperature distributions of NGV/shroud walls}

Figure 5 shows the normalized temperature contours of the NGV and shroud walls. A legend scale of $\mathrm{T}=0.5-0.875$, instead of $0.5-1.0$, is used in Figures 5-8 for clarity. The high 


\begin{tabular}{lll}
\hline & No. & Mass flow rate \\
\hline Downstream hole & 1 & $14.1 \%$ \\
Upstream holes & 1 & $12.9 \%$ \\
& 2 & $12.4 \%$ \\
& 3 & $15.1 \%$ \\
& 4 & $14.7 \%$ \\
& 5 & $15.1 \%$ \\
& 6 & $15.6 \%$ \\
\hline
\end{tabular}

Table 2. Mass flow rates through cooling holes.

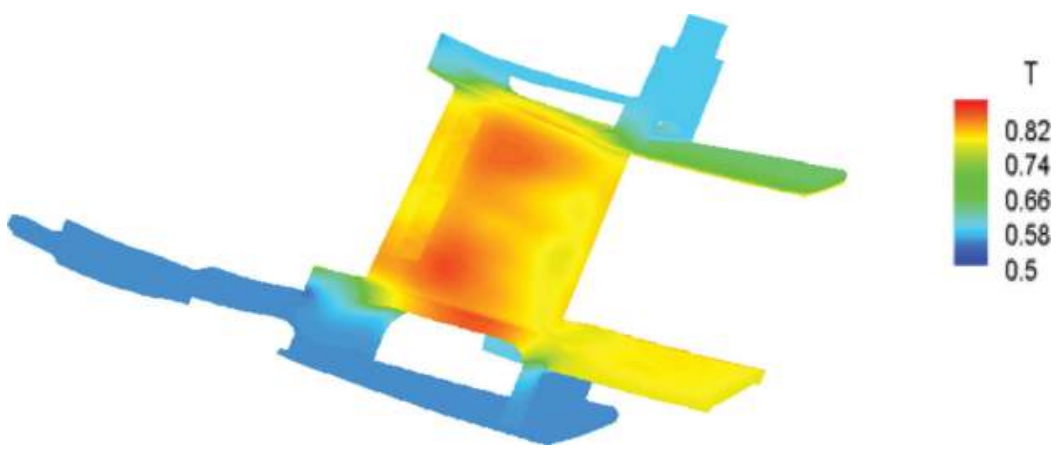

Figure 5. Temperature contours of the NGV and shrouds.

temperature is found in two local areas, one upper and one lower, on the NGV pressure side near the rectangular cooling air outlets as well as the upper middle region of the external shroud, with a maximum value of 0.87 . At the shroud outer surfaces, the wall temperature is comparatively low.

The temperature contours at the NGV external and internal surfaces are presented in Figures 6-7, respectively, and the temperature distributions over the fin surfaces are shown in Figure 8. Figures 6-8 illustrate the temperature distributions at both pressure and suction sides. At these surfaces, the temperature variation is significant. As mentioned earlier, the higher temperature occurs on the external walls of the pressure side than that on the suction side, and two local high-temperature areas around the rectangular outlets are observed on the pressure side. These findings are consistent with the temperature distributions over the NGV internal and fin surfaces These results clearly imply that the semi-empirical 1-D heat transfer analysis is not suitable for the practical complex 3-D heat transfer phenomena.

In Figure 9, the metal temperature contours at three sections with two, (a) and (b), cutting through the hot areas of the NGV pressure side, and one, (c), through the middle portion, with a temperature scale of $0.7-0.86$. The metal temperature variations at these sections are obvious and in agreement with the findings in Figures 5-8. The maximum variation of metal 

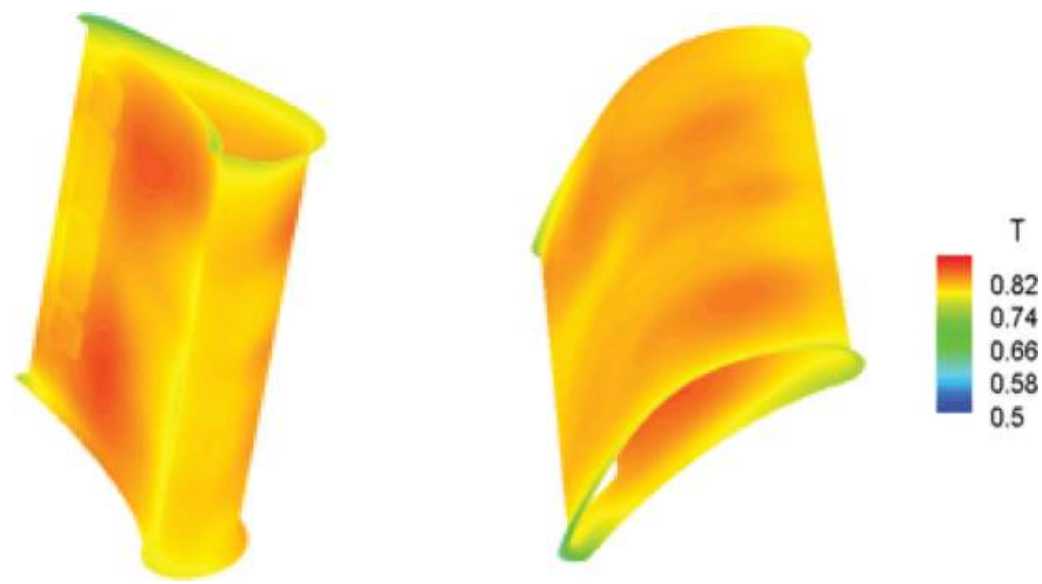

Figure 6. Temperature contours at the external surfaces of the pressure and suction sides.
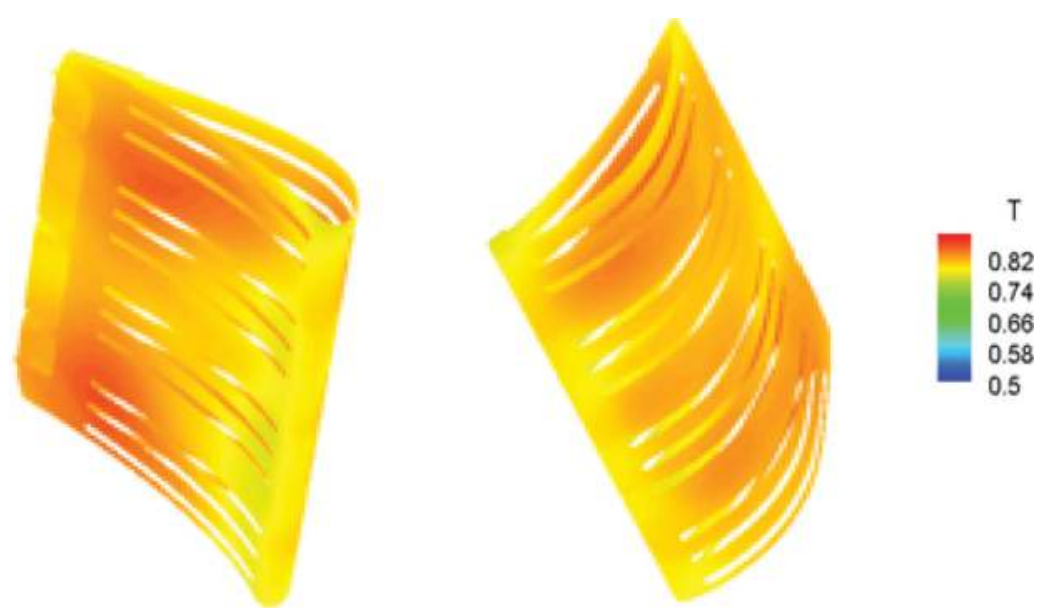

Figure 7. Temperature contours at the internal surfaces of the pressure and suction sides.

wall temperature in Figure 9 is 0.12 . The Biot number at the NGV internal/external surfaces is only 0.006 in the present heat transfer case, much less than 1 . This means that the resistance of the heat conduction in the NGV solid regions is much less than that of the heat convection through its surfaces. As a result, the temperature distribution in the NGV body is mainly determined by the complex heat convection among the NGV body, external main flow, and internal cooling flow.

The temperature distributions at the two sections across the centers of the hot spots on the pressure side observed in Figures 5-9 is shown in Figure 10, and the outlines of the air-cooling slot, seven holes, and NGV main body are included in the figure. The upper section passes 

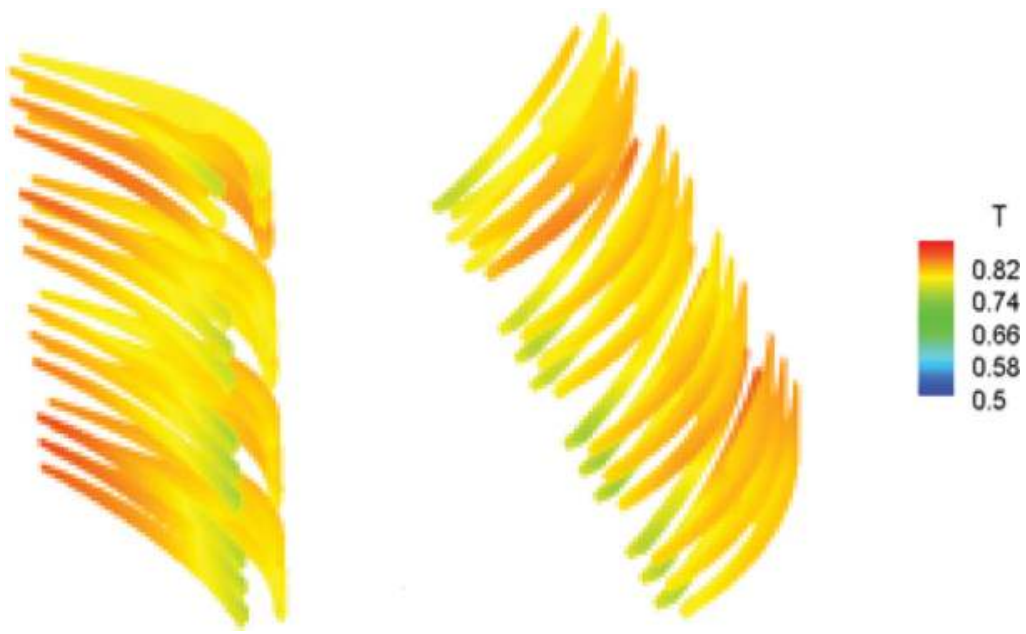

Figure 8. Temperature contours at the cooling fin surfaces.

through the middle between the two rectangular outlets of the cooling flow and does not cut through any cooling hole. The lower section just passes through the edges of a rectangular outlet and a cooling hole. It is anticipated that the cooling airflow is restricted to some extent at local areas of these two sections. This may be the reason why the temperature around these local areas is higher than the other areas. The temperature distributions at the middle section across an upstream hole and the downstream hole of the cooling slot are shown in Figure 11. This geometrical configuration may explain why the temperature at the middle of the NGV and fins is relatively lower than in the neighboring areas.

As stated before, the heat transfer is complex inside and outside the NGV/shrouds, and it is no longer governed by the semiempirical 1D convection-conduction-convection analysis, i.e., from the main hot flow to the NGV metal body and then to the cooling airflow in the cooling chamber. For instance, if only velocity or Mach number is considered (Figure 2), it is anticipated that the heat flux at the pressure side is lower than that at the suction side. However, according to the temperature contours in Figure 3, the heat flux at the pressure side tends to be higher than the suction side.

Figure 12 provides the heat flux through the NGV internal and external surfaces at the three sections, as shown in Figures 9-11. In this figure, the heat flux values are normalized by the mean maximum heat fluxes across the NGV internal and external surfaces, where the metal cross sections are shown in dark blue thick lines. The data points in red stand for the heat flux across the NGV external surface, while the green dots represent the heat flux across the internal surface. The positive sign of heat flux means the flux flows toward the cooling chamber, and for the negative sign, it is in the opposite direction. The characteristics of the heat flux patterns at the three sections are similar. Owing to the 3D nature of the NGV/shroud heat transfer, the heat fluxes across the NGV internal and external surfaces are not equal at all. Around the NGV leading edge, the heat flux across the internal surface is larger than that for 
(a)

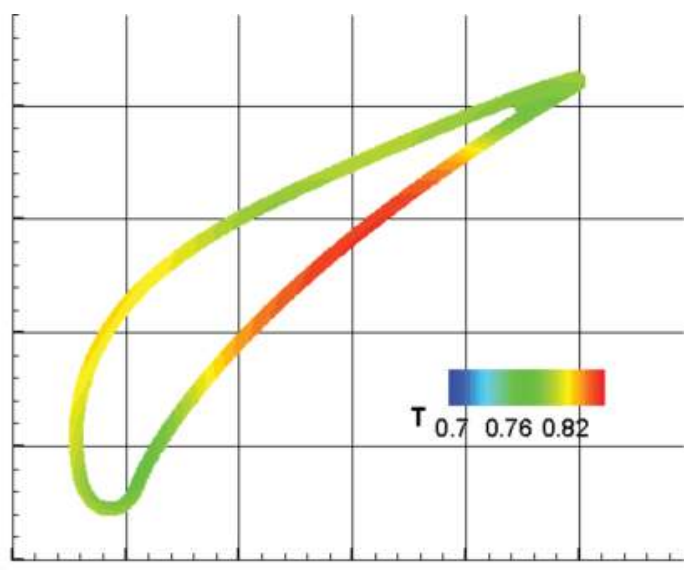

(b)

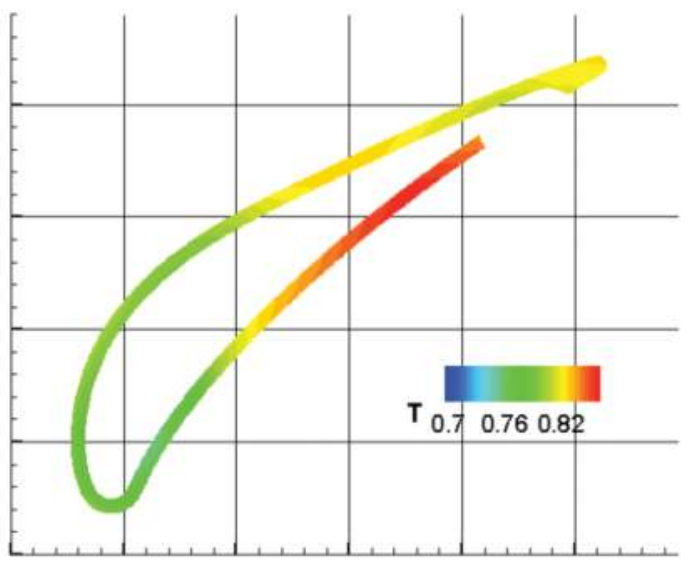

(c)

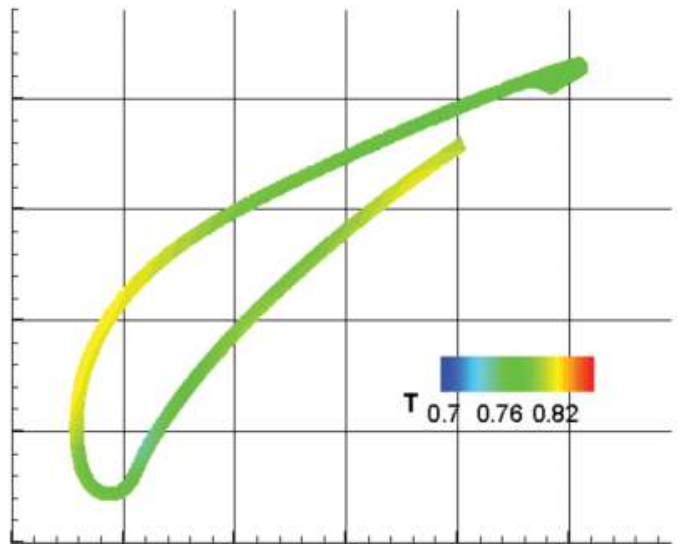

Figure 9. Metal temperature contours: (a) and (b) across hot spots and (c) across the middle of the NGV. 

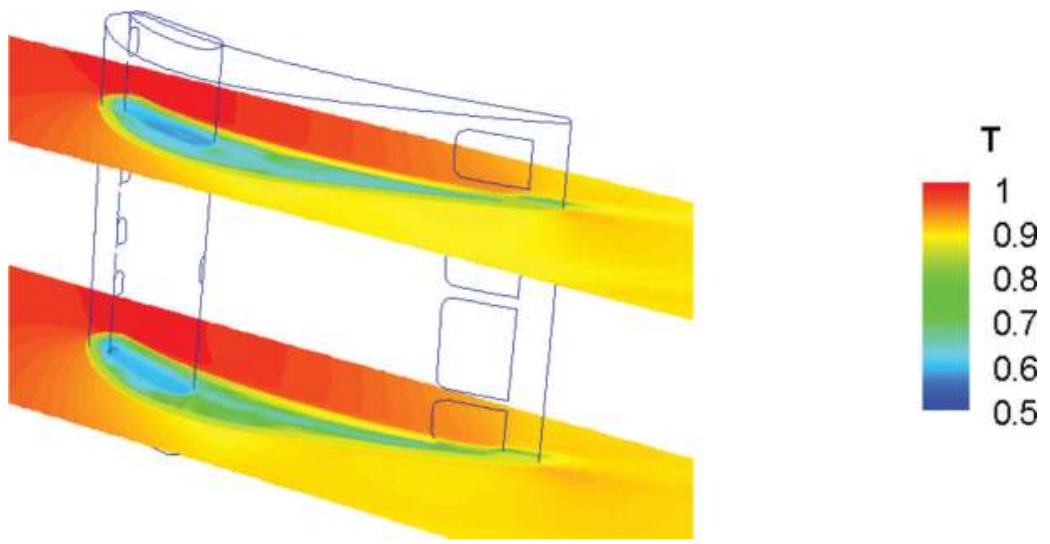

Figure 10. Temperature contours at the two sections across the high-temperature spots on the NGV external surfaces.

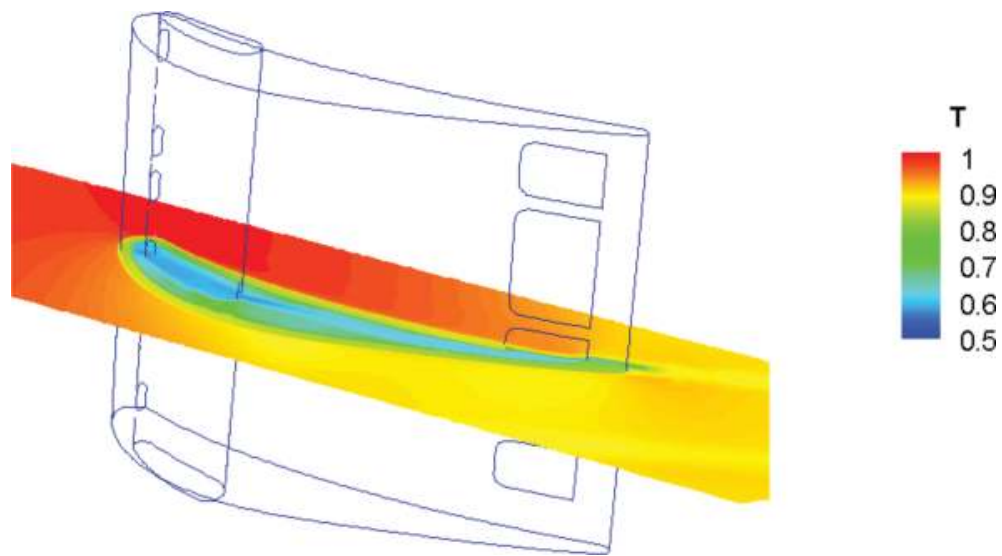

Figure 11. Temperature contours on the section across the downstream hole and an upstream hole of the cooling slot.

the external surface, in particular at the lower and middle cross sections (Figure 12(b) and (c)). Generally, the flux is high near the NGV leading edge and steadily decreases along the trailing edge. Local variations and bumps of the heat flux profiles are observed, which are determined by the local flow conditions and geometry.

Around the trailing edge, there is a substantial negative heat flux at the external surface. Note that the area with negative flux is a portion of or close to the cooling air exiting passage (Figure 1) and the cooling air runs over the tiny trailing edge region. Displayed in Figure 13 are the flow/metal temperature distributions around the NGV trailing edge at these three sections: (a) for the upper section, (b) for the lower section, and (c) for the middle section. It is obvious that the flow temperature around the trailing edge region is lower than the neighboring metal temperature at all three cross sections. Therefore, the heat flux alters its direction, i.e., the negative heat flux is found at these small regions. 
(a)

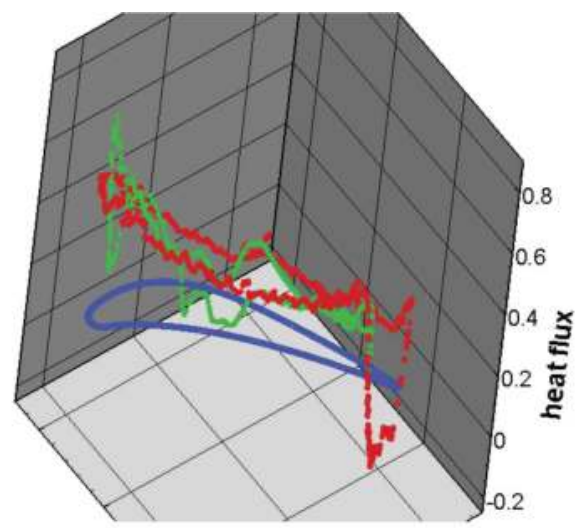

(b)

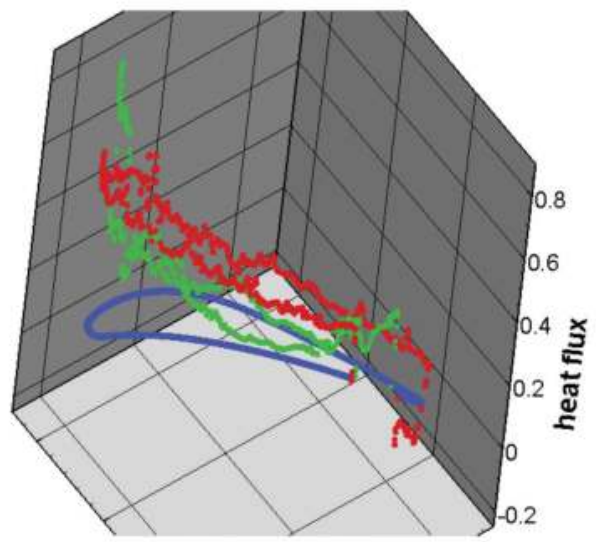

(c)

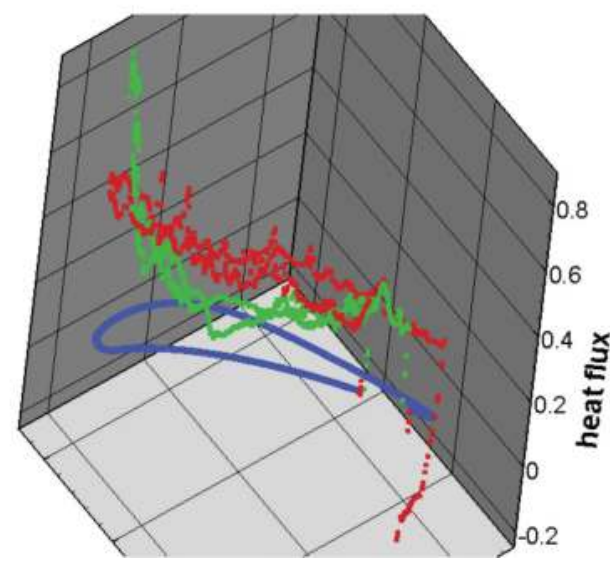

Figure 12. Normalized heat flux (red for external surface and green for internal surface): (a) at the upper section, (b) at the lower section, and (c) at the middle section. 
(a)

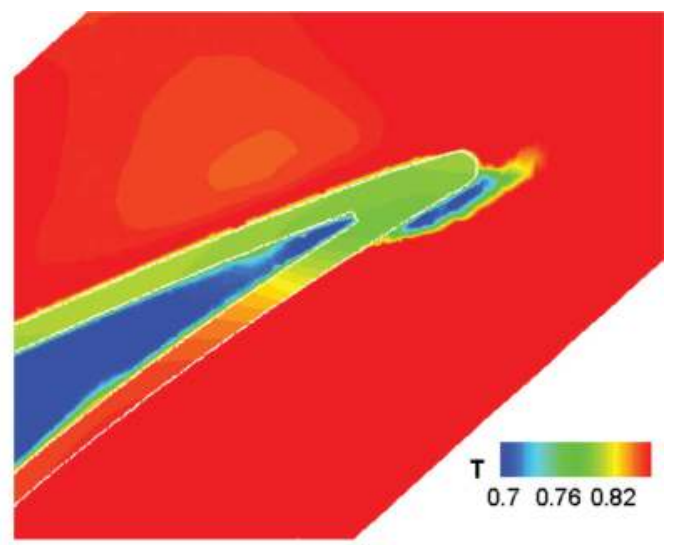

(b)

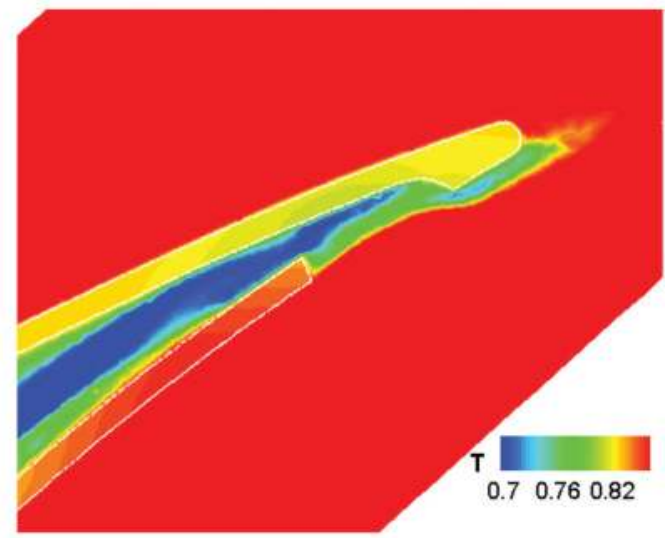

c)

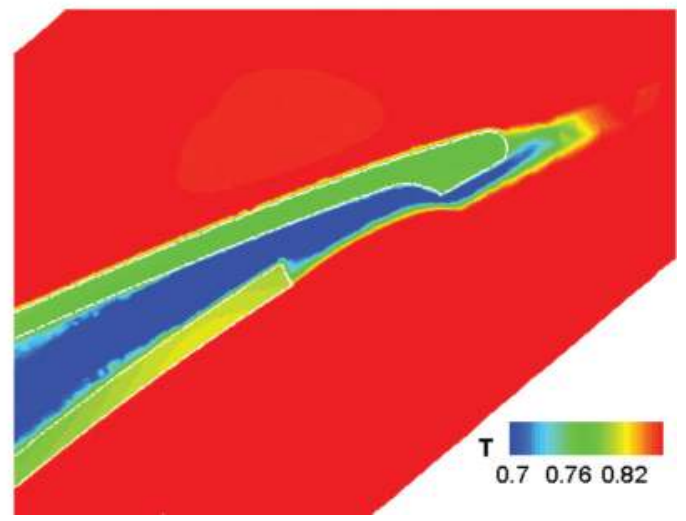

Figure 13. Temperature contours near the NGV trailing edge: (a) at the upper section, (b) at the lower section, and (c) at the middle section. 
(a)

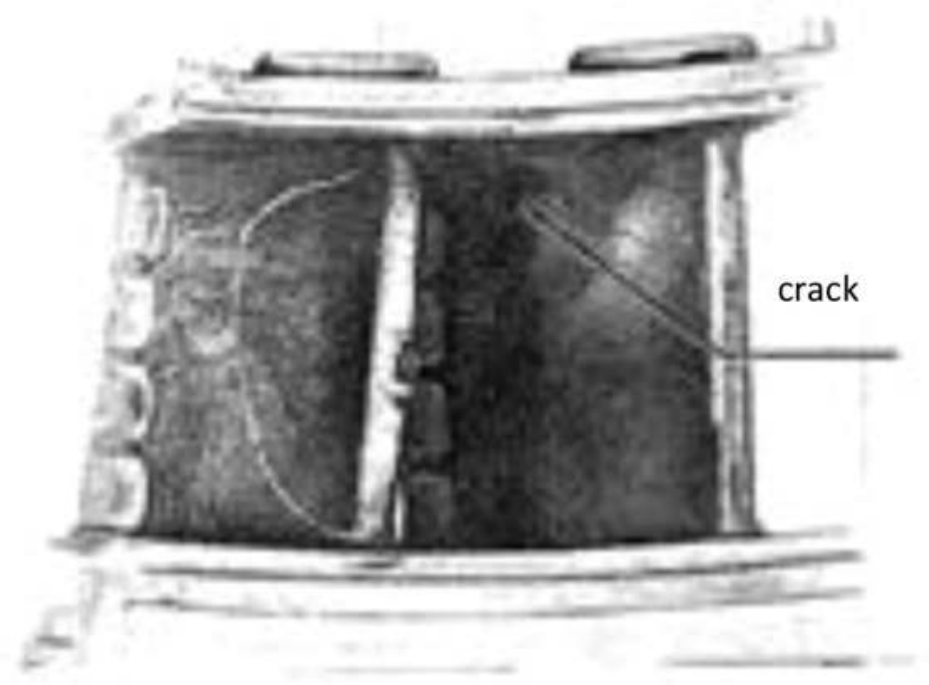

(b)

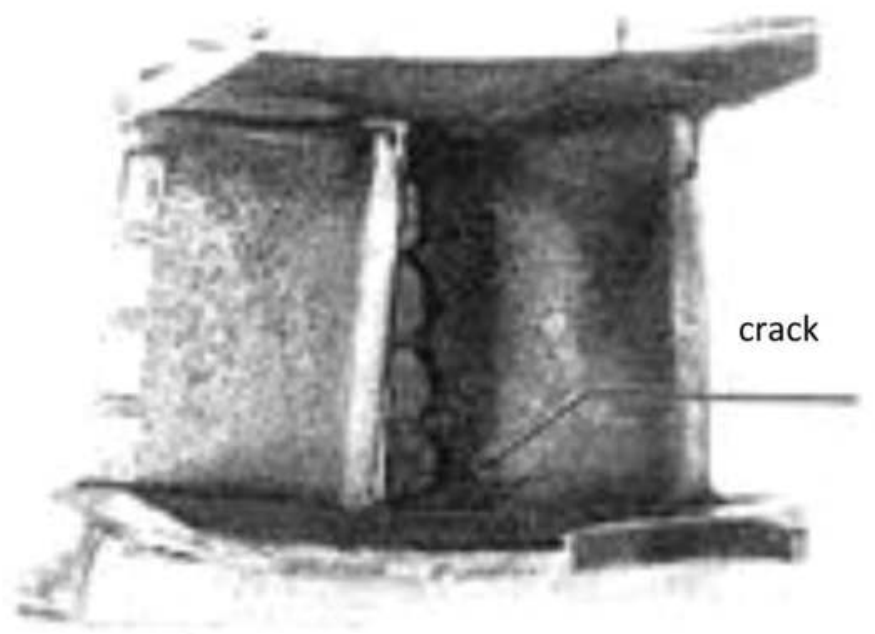

Figure 14. Cracks at the upper and lower portions of the NGV pressure side, starting from the rectangular cooling air exits.

There are many factors affecting the complex heat transfer phenomena observed in Figure 13: the external flow parameters (local gas properties, pressure, temperature, and velocity), the cooling flow variables (local air properties, pressure, temperature, and velocity), and the local geometries of the NGV and cooling flow passage (shape and location of the cooling holes, 
outlets, fins, shrouds, etc.). Moreover, as shown in Figures 1, 2-4, 7-8, and 10-11, the cooling air enters the cooling chamber through the internal shroud, crosses the seven cooling holes, flows over the 24 cooling fins, and eventually exits the chamber through four rectangular outlets. It is expected that complex secondary flows can be generated because of the jet flows issuing from the cooling holes and the interactions among the cooling flow and cooling fins [12]. The detailed analysis of the secondary flow effect is outside of the scope of the present study, and it can play an important role in the NGV cooling [6].

The photos of damaged NGVs after a certain service time are illustrated in Figure 14, and there are cracks observed at the pressure side. A long crack from the rectangular outlet edge to the NGV leading edge is located at the upper portion of the NGV body as shown in Figure 14(a), and a short crack also beginning from the rectangular outlet edge occurs at the NGV lower surface shown in Figure 14(b). These field observations are in good agreement with the above-predicted results. As shown in Figure 9, the cross sections (a) and (b) are located in the upper and lower regions of the NGV body, close to or agreeing with the crack locations. The high metal temperature area is found from the middle of the span to the cooling rectangular outlet on the NGV pressure side. The high temperature decreases the metal strength, and the rectangular geometry and temperature variation increase local metal material stress. Consequently, these rectangular edges in the upper and lower regions at the pressure side are more vulnerable and prone to cracking.

As a technical demonstration case, the obtained results provide essential information for the design or life analysis of the NGV/shroud assembly. Moreover, the results recommend that the cooling flow can be optimized by rearranging the holes, outlets of the cooling flow passage, and/or altering the numbers and sizes of cooling holes. It is clear that the detailed heat transfer analysis of the NGV/shroud assembly helps to achieve this goal.

\section{Conclusions}

In this chapter, the flow field and conjugate heat transfer of an internally air-cooled nozzle guide vane and shroud assembly of a gas turbine engine at flight conditions are numerically studied. The high-fidelity CFD model is created, and the simulations are performed with properly defined boundary conditions. The characteristics of the complex flow and temperature fields are discussed. The Mach number is higher, and the temperature is lower on the NGV suction side than those on the pressure side. The results reveal that the NGV surface temperature varies considerably and the thermal stress could be excessively high. Two hightemperature spots on the NGV pressure side are observed, which can significantly reduce the NGV service life. These findings are closely correlated with the locations of the cooling holes and outlets of the cooling flow passage, and consistent with the field observations of damaged NGVs. As a technology demonstration case, the findings provide essential data for the design or service life assessment of the NGV/shrouds, for example, improving the cooling effectiveness. 


\section{Acknowledgements}

The authors are grateful to the Air Defence System Program, National Research Council of Canada for supporting this research project. The authors would also like to express their thanks to Dr. Francois Fortin for the valuable comments and suggestions during the preparation of this manuscript.

\section{Author details}

Lei-Yong Jiang*, Yinghua Han and Prakash Patnaik

*Address all correspondence to: lei-yong.jiang@nrc-cnrc.gc.ca

Aerospace, National Research Council of Canada, Ottawa, Ontario, Canada

\section{References}

[1] Lefebvre AH, Ballal DR. Gas Turbine Combustion. New York: Taylor and Francis Group; 2010

[2] Mazur Z, Hernandez-Rossette A, Garcia-Illescas R, Luna-Ramirez A. Analysis of conjugate heat transfer of a gas turbine first stage nozzle. Applied Thermal Engineering. 2006;26:1796-1806

[3] Charbonnier D, Ott P, Jonsson M, Kobke T, Cottier F. Comparison of Numerical Investigations with Measured Heat Transfer Performance of a Film Cooled Turbine Vane. ASME IGTI Paper, GT2008-50623.2008

[4] Heidmann JD, Kassab AJ, Divo EA, Franklin Rodriguez F, Steinthorsson E. Conjugate Heat Transfer Effects on a Realistic Film-Cooled Turbine Vane. ASME IGTI Paper, GT2003-38553. 2003

[5] Jiang LY, Manipurath S, Bourque G, Houde M. Flow-field of a triple-walled gas-sampling probe with sub-cooled boiling effect. Flow Measurement and Instrumentation. 2005;18:156-116

[6] Harsqama SP, Burton CD, Chana KS. Measurements and computations of external heat transfer and film cooing in turbines. Proceedings of the 10th International Symposium on Air Breathe Engines (ISABE). 1991. pp. 1276-1284

[7] Ferziger JH, Peric M. Computational Methods for Fluid Dynamics. New York: SpringerVerlag; 2000

[8] ANSYS Fluent, Fluent 15 Documentation. Lebanon, NH, USA. 2015

[9] Menter FR. Two-equation Eddy-viscosity turbulence models for engineering applications. AIAA Journal. 1994;32(8):1598-1605 
[10] Jiang LY, Corber A. Assessment of combustor working environments. International Journal of Aerospace Engineering. 2012;2012:6. Article ID: 217463. DOI: 10.1155/2012/ 217463

[11] Snedden G, Roos T, Naidoo K. Detailed disc assembly temperature prediction: Comparison between CFD and simplified engineering methods. ISABE Paper 2005-1130. 2005

[12] Samimy M, Breuer KS, Leal LG, Steen PH. A Gallery of Fluid Motion. UK: Cambridge University Press; 2003 
